\title{
Optimal Sensor Placement for Linear Decentralized Estimation with Coherent and Orthogonal Multiple Access Channels
}

\author{
Eduard A. Jorswieck and Martin Mittelbach \\ Communications Theory, Communications Laboratory \\ Dresden University of Technology, 01062 Dresden, Germany \\ Email: \{jorswieck,mittelbach\}@ifn.et.tu-dresden.de, Web: http://www.ifn.et.tu-dresden.de/tnt
}

\begin{abstract}
The design of wireless sensor networks requires cross layer optimization of sensing, signal processing and communication. We consider the linear decentralized estimation model in which low complex sensors sense, amplify, and forward the noisy and scaled observation of a source variable via a multipleaccess channel (MAC) to the fusion center (FC), where the FC has perfect channel state information. We study the optimal placement of sensors located between the source and the FC. Using majorization, we prove for the orthogonal MAC, that an equidistant placement of the sensors between source and FC is optimal. For the general coherent MAC, the same behavior is explained and observed. Bounds on the loss due to suboptimal sensor placement are derived. The clear behavior changes if the outage probability is used for sensor placement. At high SNR, the average distortion is Schur-convex while for small SNR, it is Schur-concave. Numerical simulations illustrate the theoretical findings.
\end{abstract}

\section{INTRODUCTION, SYSTEM MODELS, AND PROBLEM STATEMENTS}

We consider the scenario of a generic wireless sensor network in which noisy observations of a source are measured by multiple sensors. The low-cost sensors amplify the received signal and send it to a fusion center (FC), where an estimate of the realization of the source is build [1]. Three main operations - sensing, processing and communication - should be jointly optimized in a wireless sensor network since the resources are limited. Sensors are limited in terms of computational power, bandwidth, and energy [2].

\section{A. Recent results and contributions}

The real valued sensor messages are analog transmitted to the FC. We follow the system model in [3], which is based on amplify-and-forward operations at the sensors and study the orthogonal multiple-access channel (MAC) as well as the coherent MAC. The FC builds an estimate of the source symbol using the linear minimum mean-square error (MMSE) receiver. The performance of the reconstruction is measured by the average distortion. In contrast to recent work, the sensors have individual power constraints and we consider the problem of the sensor placement in the space between source and FC.

The sensor placement problem in wireless sensor networks is studied recently in a number of works (see the survey in

This research was supported in part by the Deutsche Forschungsgemeinschaft (DFG) under grant JO 801/3-1.
[4]). In [5], the sampling in space and time is considered and a characterization of the optimal sensor placement is derived. In [6], the average transmit power is minimized with finiterate feedback for coherent communication in a wireless sensor network.

Our contributions are as follows: For orthogonal and coherent MAC, we answer the question of how to place a set of sensors between the source and the FC by a three step approach using majorization theory. First, the impact of random channel gains on the average distortion for fixed sensor gains is derived. The average distortion is Schur-convex with respect to the average channel gains, i.e., the minimum average distortion is achieved for symmetrically distributed sensors around the FC. Second, the impact of random sensor gains on the average distortion for fixed channel gains is analyzed. The behavior is identical to the first case with sensor and channel gains switching their roles. In the final third step, these results are combined to conclude that the minimum average distortion with random channel and sensor gains is achieved if the sensors are placed equidistant from source and FC. A brief discussion of the outage probability shows that for high SNR the optimal sensor placement does not change whereas for small SNR (high outage probability), the results change completely. Numerical simulations illustrate the results.

\section{B. System models}

We follow the model in [3] and consider the case where $L$ sensors $S_{1}, \ldots, S_{L}$ collect scaled and noisy realizations $s$ of a random variable $\mathrm{S}$. Sensor $S_{l}$ observes

$$
x_{l}=h_{l} s+n_{l},
$$

with $h_{l}$ denoting a random source-to-sensor channel gain and $n_{l}$ representing zero-mean complex Gaussian noise with variance $\sigma^{2}$. For later use we define $\rho=\frac{1}{\sigma^{2}}$. The noise at all sensors is assumed to be i.i.d. (independent and identically distributed). As in [7] we model with the source-to-sensor channel gain the uncertainty in some physical aspect of the sensing process. In the rest of the paper we abbreviatory use the term sensor gain. The sensors amplify the noisy observations and transmit it to the FC over a MAC. This MAC can be thought of as a MAC in one cell in the model of [2]. The FC forms an estimate $\hat{s}$ of the observation $s$. 


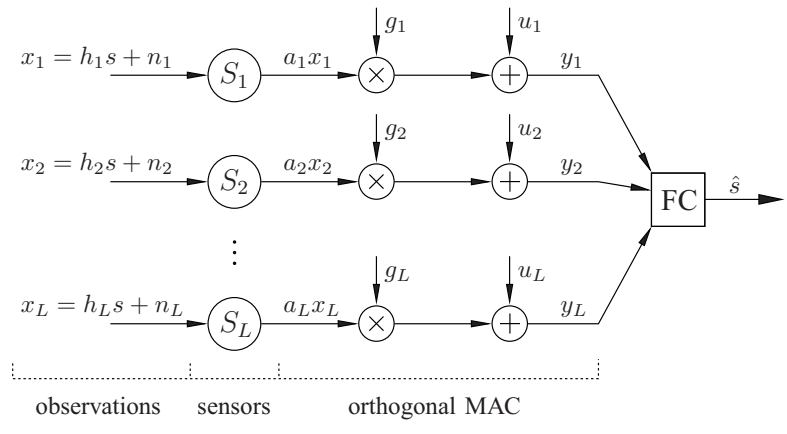

Fig. 1. System model with orthogonal MAC.

We consider two different MAC models. Both assume perfect channel state information (CSI) at the fusion center. The first is additionally an orthogonal MAC in which the transmission of the sensors are scheduled orthogonally in time or frequency. The second is a standard MAC in which all transmissions take place simultaneously. Note that for the first setting, coordination between the sensors is needed, whereas for the second the sensors transmit anytime. However, we assume symbol synchronous transmission.

The system model with the orthogonal MAC is shown in Fig. 1. For the orthogonal MAC, the received signals at the FC are given by

$$
y_{l}=g_{l} a_{l} h_{l} s+g_{l} a_{l} n_{l}+u_{l}, \quad l=1, \ldots, L,
$$

with $a_{l}$ denoting the sensor amplification factor and $g_{l}$ the random sensor-to-FC channel gain of sensor $S_{l}$. The sensor-toFC channel gain models the wireless fading channel between sensor and FC. We will refer to this gain subsequently as channel gain. Furthermore, in (1) the quantity $u_{l}$ represents zero-mean complex Gaussian noise with variance $\sigma^{2}$. The noise again is assumed to be i.i.d. ${ }^{a}$

The linear MMSE estimator at the FC results in an achievable MSE $D_{\mathrm{o}}$ satisfying [8]

$$
\frac{1}{D_{\mathrm{o}}}=1+\sum_{l=1}^{L} \frac{g_{l}^{2} a_{l}^{2} h_{l}^{2}}{1 / \rho+g_{l}^{2} a_{l}^{2}} .
$$

Note that the average transmit power of sensor $S_{l}$ is given by $P_{l}=a_{l}^{2}\left(h_{l}^{2}+1\right)$.

The system model with the coherent MAC is shown in Fig. 2. For the coherent MAC, the received signal at the FC is given by

$$
y=\sum_{l=1}^{L} g_{l} a_{l} h_{l} s+\sum_{l=1}^{L} g_{l} a_{l} n_{l}+u .
$$

with $a_{l}$ and $g_{l}$ as above and $u$ being zero-mean complex Gaussian noise with variance $\sigma^{2}$. The linear MMSE estimator at the $\mathrm{FC}$ results in an achievable MSE $D_{\mathrm{c}}$ given by [3, eq. (6)]

$$
\frac{1}{D_{\mathrm{c}}}=1+\frac{\left(\sum_{l=1}^{L} g_{l} h_{l} a_{l}\right)^{2}}{1 / \rho+\sum_{l=1}^{L} g_{l}^{2} a_{l}^{2}} .
$$

\footnotetext{
a We choose the variance of the noise at the FC identical to the variance of the noise at the sensors to simplify analysis. However, it is trivial to extend the model and the analysis for different variances.
}

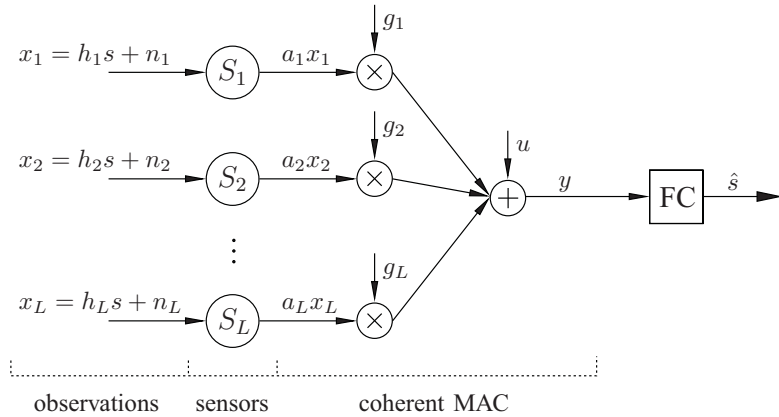

Fig. 2. System model with coherent MAC.

Assuming individual power constraints at the sensors, i.e. $P_{l}=$ 1 , leads to normalized amplification factors

$$
a_{l}^{2}=\left(h_{l}^{2}+1\right)^{-1} \text {. }
$$

Define $x_{l}=\left(1+h_{l}^{2}\right)^{-1}$ to obtain for the achievable inverse MSEs $D_{\mathrm{o}}$ and $D_{\mathrm{c}}$

$$
\frac{1}{D_{\mathrm{o}}}=1+\sum_{l=1}^{L} \frac{g_{l}^{2} \frac{h_{l}^{2}}{1+h_{l}^{2}}}{1 / \rho+g_{l}^{2} \frac{1}{1+h_{l}^{2}}}=1+\sum_{l=1}^{L} \frac{g_{l}^{2}\left(1-x_{l}\right)}{1 / \rho+g_{l}^{2} x_{l}}
$$

and

$$
\frac{1}{D_{\mathrm{c}}}=1+\frac{\left(\sum_{l=1}^{L} g_{l} \sqrt{\frac{h_{l}^{2}}{1+h_{l}^{2}}}\right)^{2}}{1 / \rho+\sum_{l=1}^{L} g_{l}^{2} \frac{1}{1+h_{l}^{2}}}=1+\frac{\left(\sum_{l=1}^{L} g_{l} \sqrt{1-x_{l}}\right)^{2}}{1 / \rho+\sum_{l=1}^{L} g_{l}^{2} x_{l}}
$$

For $\rho \rightarrow \infty$ (high SNR) the following limits are obtained:

$$
\frac{1}{D_{\mathrm{o}}} \underset{\rho \rightarrow \infty}{\longrightarrow} 1+\sum_{l=1}^{L}\left(\frac{1}{x_{l}}-1\right)=1+\sum_{l=1}^{L} h_{l}^{2}
$$

and

$$
\frac{1}{D_{\mathrm{c}}} \underset{\rho \rightarrow \infty}{\longrightarrow} 1+\frac{\left(\sum_{l=1}^{L} g_{l} \sqrt{1-x_{l}}\right)^{2}}{\sum_{l=1}^{L} g_{l}^{2} x_{l}}
$$

which is a constant larger than or equal to (7).

\section{Problem statements}

In this work, we want to understand the impact of the sensor location on the distortion. Recall, in (5) and (6) we denote by $h_{1}, \ldots, h_{L}$ the sensor gains and by $g_{1}, \ldots, g_{L}$ the channel gains.

1) Fixed sensor gains, random channel gains: First, we consider the scenario in which the sensor gains are fixed $h_{1}=$ $h_{2}=\ldots h_{L}=1$ which leads to

$$
x_{l}=\left(h_{l}+1\right)^{-1}=1 / 2 .
$$

Furthermore, let the random channel gains $g_{1}, \ldots, g_{L}$ be modeled as $g_{l}^{2}=c_{l} w_{l}$ with $w_{1}, \ldots, w_{L}$ being i.i.d. standard exponentially distributed. The resulting average distortion is

$$
\overline{D_{\mathrm{o}}}(\boldsymbol{c})=\mathbb{E}_{w}\left[D_{\mathrm{o}}\right]=\mathbb{E}_{w}\left[\left(1+\sum_{l=1}^{L}\left(1-\frac{2}{2+\rho c_{l} w_{l}}\right)\right)^{-1}\right]
$$


and

$\overline{D_{\mathrm{c}}}(\boldsymbol{c})=\mathbb{E}_{w}\left[D_{\mathrm{c}}\right]=\mathbb{E}_{w}\left[\left(1+\frac{\rho\left(\sum_{l=1}^{L} \sqrt{c_{l} w_{l}}\right)^{2}}{2+\rho \sum_{l=1}^{L} c_{l} w_{l}}\right)^{-1}\right]$.

Problem 1: What is the impact of the average channel gains $\boldsymbol{c}=\left[c_{1}, \ldots, c_{L}\right]$ on the average distortion?

2) Random sensor gains, fixed channel gains: Next, we consider the scenario in which the channel gains are fixed and normalized, i.e. $g_{1}=g_{2}=\ldots=g_{L}=1$, and the sensor gains $h_{1}, \ldots, h_{L}$ are random. The sensor gains are modeled as $h_{l}^{2}=d_{l} v_{l}$ with $v_{1}, \ldots, v_{L}$ being i.i.d. random variables with expectation equal to one. Note that we do not need to consider a specific distribution of the random variables $v_{l}$ for the subsequent analysis. We obtain

$$
\frac{1}{D_{\mathrm{o}}}=1+\sum_{l=1}^{L} \frac{\left(1-x_{l}\right)}{1 / \rho+x_{l}}=1+\sum_{l=1}^{L} \frac{\rho h_{l}^{2}}{1+\rho+h_{l}^{2}} .
$$

The resulting average distortion for the orthogonal MAC is

$$
\overline{D_{\mathrm{o}}}(\boldsymbol{d})=\mathbb{E}_{v}\left[D_{\mathrm{o}}\right]=\mathbb{E}_{v}\left[\left(1+\sum_{l=1}^{L} \frac{\rho d_{l} v_{l}}{1+d_{l} v_{l}+\rho}\right)^{-1}\right] \text {. }
$$

For the coherent MAC the resulting average distortion is

$$
\overline{D_{\mathrm{c}}}(\boldsymbol{d})=\mathbb{E}_{v}\left[D_{\mathrm{c}}\right]=\mathbb{E}_{v}\left[\left(1+\frac{\left(\sum_{l=1}^{L} \sqrt{\frac{d_{l} v_{l}}{1+d_{l} v_{l}}}\right)^{2}}{1 / \rho+\sum_{l=1}^{L} \frac{1}{1+d_{l} v_{l}}}\right)^{-1}\right] .
$$

Problem 2: What is the impact of the average sensor gains $\boldsymbol{d}=\left[d_{1}, \ldots, d_{L}\right]$ on the average distortion?

3) Random sensor and channel gains: Finally, we consider the case in which the channels as well as the sensor gains are random. However, the average channel gains and sensor gains are not independent. If a sensor is close to the source it is automatically far from the FC and vice versa. We model this relationship by

$$
h_{l}^{2}=d_{l} v_{l} \quad \text { and } \quad g_{l}^{2}=\overbrace{\left(1-d_{l}\right)}^{c_{l}} w_{l}, \quad l=1, \ldots, L .
$$

The small scale fading realizations $v_{1}, \ldots, v_{L}, w_{1}, \ldots, w_{L}$ are distributed as in the models above. From (14) it follows that $\sum_{l=1}^{L} d_{l}+\sum_{l=1}^{L} c_{l}=\sum_{l=1}^{L} c_{l}+\sum_{l=1}^{L}\left(1-c_{l}\right)=L$

Obviously, this is an idealized model. However, it includes many cases of practical interest and allows an analytical approach. We define the composed vector $\gamma=[\boldsymbol{d}, \boldsymbol{c}]$ and consider the average distortion for the orthogonal MAC

$$
\overline{D_{\mathrm{o}}}(\gamma)=\mathbb{E}_{w, v}\left[\left(1+\sum_{l=1}^{L} \frac{c_{l} w_{l} \frac{d_{l} v_{l}}{1+d_{l} v_{l}}}{1 / \rho+c_{l} w_{l} \frac{1}{1+d_{l} v_{l}}}\right)^{-1}\right]
$$

and for the coherent MAC

$$
\overline{D_{\mathrm{c}}}(\gamma)=\mathbb{E}_{w, v}\left[\left(1+\frac{\left(\sum_{l=1}^{L} \sqrt{c_{l} w_{l} \frac{d_{l} v_{l}}{1+d_{l} v_{l}}}\right)^{2}}{1 / \rho+\sum_{l=1}^{L} c_{l} w_{l} \frac{1}{1+d_{l} v_{l}}}\right)^{-1}\right] .
$$

Problem 3: What is the impact of the sensor placement $\gamma$ on the average distortion?

\section{PRELIMINARIES}

In order to compare different scenarios, we will apply majorization theory. Without loss of generality, we order the sensors in a decreasing way according to their average sensor gains, i.e. $c_{1} \geq c_{2} \geq \ldots \geq c_{L}$. The constraint regarding the sum of these gains verifies that we compare scenarios in which the sensors obtain the same average sum power. We need the following definitions [9].

Definition 2.1: For two vectors $\boldsymbol{x}, \boldsymbol{y} \in \mathbb{R}^{n}$ we say that the vector $\boldsymbol{x}$ majorizes the vector $\boldsymbol{y}$ and write $\boldsymbol{x} \succ \boldsymbol{y}$ if

$$
\sum_{k=1}^{m} x_{k} \geq \sum_{k=1}^{m} y_{k}
$$

for $m=1, \ldots, n-1$ and $\sum_{k=1}^{n} x_{k}=\sum_{k=1}^{n} y_{k}$.

The next definition describes a function $\Phi$ which is applied to the vectors $\boldsymbol{x}$ and $\boldsymbol{y}$ with $\boldsymbol{x} \succ \boldsymbol{y}$.

Definition 2.2: A real-valued function $\Phi$ defined on $\mathcal{A} \subset$ $\mathbb{R}^{n}$ is said to be Schur-convex on $\mathcal{A}$ if from $\boldsymbol{x} \succ \boldsymbol{y}$ on $\mathcal{A}$ follows $\Phi(\boldsymbol{x}) \geq \Phi(\boldsymbol{y})$. Similarly, $\Phi$ is said to be Schurconcave on $\mathcal{A}$ if from $\boldsymbol{x} \succ \boldsymbol{y}$ on $\mathcal{A}$ follows $\Phi(\boldsymbol{x}) \leq \Phi(\boldsymbol{y})$.

Schur-convexity and Schur-concavity correspond to our understanding of less and more spread out. The most spread out vector has equal entries, while the less spread out vector has only one entry which is equal to $L$ in our case.

The operational meaning of majorization in our wireless sensor network context seems first unmotivated if the two separate cases with fixed channel gains or fixed sensor gains are studied. However, in the case in which random channel gains as well as random sensor gains are considered, the operational meaning is explained in the following two figures.

In Fig. 3, the black sensors are symmetrically arranged and have equal distance to the source as well as to the FC. This implies $\boldsymbol{d}=\frac{1}{2}[1,1, \ldots, 1]=\boldsymbol{c}$. This situation is illustrated in contrast to the case, where some sensors are closer (gray) to the source and others are closer to the FC (white).

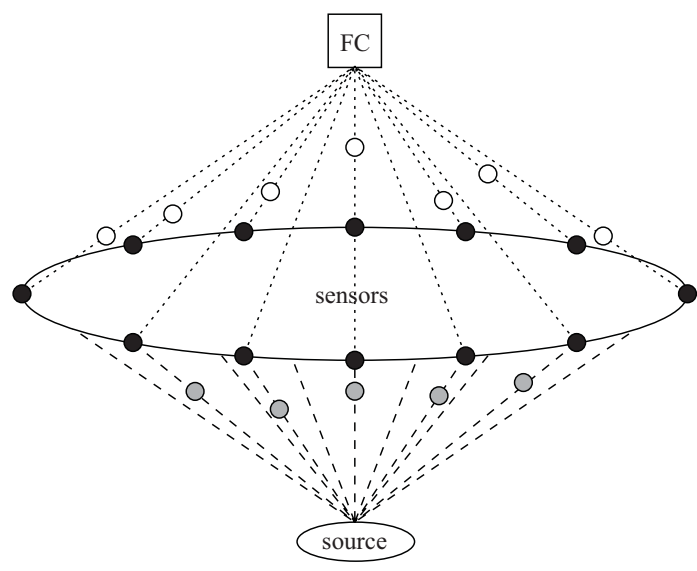

Fig. 3. Symmetric sensor placement (black, equidistant from source and FC) vs. asymmetric sensor placement (white sensors moved closer to FC, gray sensors moved closer to source).

In conclusion, this illustrates that the most spread out case corresponds to the scenario in which all sensors have the same distance to the source. The less spread out case corresponds to 
the scenario in which one sensor is very close while the others are far away. The monograph [10] gives details on majorization theory and applications in multiple antenna and multiuser networks. Before proceeding to the distortion analysis, we give some auxiliary results.

Theorem 2.1 (Theorem in [11]): Let $X=\left(X_{1}, \ldots, X_{n}\right)$ be a random vector with support $\mathcal{A}$ and interchangeable components and let $\boldsymbol{a}=\left(a_{1}, \ldots, a_{n}\right)$ and $\boldsymbol{b}=\left(b_{1}, \ldots, b_{n}\right)$ be vectors such that $\boldsymbol{a} \succ \boldsymbol{b}$. If $\phi$ is a real, continuous and convex function on $\mathcal{A}$ and symmetric in its $n$ arguments, then

$$
\mathbb{E}_{X} \phi\left(a_{1} X_{1}, \ldots, a_{n} X_{n}\right) \geq \mathbb{E}_{X} \phi\left(b_{1} X_{1}, \ldots, b_{n} X_{n}\right) .
$$

If $\phi$ is strictly convex, equality occurs only when $\boldsymbol{a}=\boldsymbol{b}$, possibly after reordering components, or $X_{i}$ is zero with probability one.

We need another characterization in terms of the cumulative probability function stated next. The random variables $w_{1}, \ldots, w_{n}$ are again i.i.d. standard exponentially distributed.

Theorem 2.2: Suppose that the inverse function $f^{-1}$ of $f$ exists and $f$ is non-negative. Further assume that the vectors $\boldsymbol{\mu}^{1}=\left(\mu_{1}^{1}, \ldots, \mu_{n}^{1}\right)$ and $\boldsymbol{\mu}^{2}=\left(\mu_{1}^{2}, \ldots, \mu_{n}^{2}\right)$ satisfy $\boldsymbol{\mu}^{1} \succ \boldsymbol{\mu}^{2}$. If $f^{-1}(x) \geq 2$ then

$$
\mathbb{P}\left[f\left(\sum_{k=1}^{n} \mu_{k}^{1} w_{k}\right) \leq x\right] \leq \mathbb{P}\left[f\left(\sum_{k=1}^{n} \mu_{k}^{2} w_{k}\right) \leq x\right] .
$$

If $f^{-1}(x) \leq 1$ then

$$
\mathbb{P}\left[f\left(\sum_{k=1}^{n} \mu_{k}^{1} w_{k}\right) \leq x\right] \geq \mathbb{P}\left[f\left(\sum_{k=1}^{n} \mu_{k}^{2} w_{k}\right) \leq x\right] .
$$

The result says that $\mathbb{P}\left[f\left(\sum_{k=1}^{n} \mu_{k} w_{k}\right) \leq x\right]$ is Schur-concave for $x \geq f(2)$ and Schur-convex for $x \leq f(1)$. The proof can be found in [12]. In the interval $f(1)<x<f(2)$ there is no clear behavior with respect to majorization.

\section{AVERAGE DISTORTION ANALYSIS}

We approach the three problems formulated above using majorization theory. For the orthogonal MAC, Schur-convexity of the average distortion is shown. For the coherent MAC, we provide arguments for the observation that the average distortion is Schur-convex as well.

\section{A. Fixed sensor gains, random channel gains}

We apply majorization to the average distortion defined in (9) and (10) for the orthogonal and coherent MAC, in order to show the impact of the average channel gains.

Theorem 3.1: The average distortion $\overline{D_{\mathrm{O}}}$ in (9) is Schurconvex with respect to average channel gain vector $c$. This implies that the sensors should be spread out uniformly/symmetrically around the FC.

Proof: The average distortion measure $\overline{D_{\mathrm{O}}}(\boldsymbol{c})$ is obviously a symmetric function and $w_{1}, \ldots, w_{L}$ are interchangeable random variables. To show convexity, we parametrize the vector

$$
\boldsymbol{c}(t)=t \boldsymbol{p}+(1-t) \boldsymbol{q}
$$

for arbitrary $\boldsymbol{p}=\left(p_{1}, \ldots, p_{L}\right) \geq 0, \quad \boldsymbol{q}=\left(q_{1}, \ldots, q_{L}\right) \geq 0$ (component-wise) with $t \in[0,1]$. Next, consider the parametrized average distortion as

$\overline{D_{\mathrm{o}}}(\boldsymbol{c}(t))=\mathbb{E}_{w}\left[\left(1+\sum_{l=1}^{L}\left(1-\frac{2}{2+\rho\left\{t p_{l}+(1-t) q_{l}\right\} w_{l}}\right)\right)^{-1}\right]$.

The second derivative of (18) with respect to $t$ is given by

$$
\frac{\partial^{2} \overline{D_{\mathrm{o}}}(t)}{\partial t^{2}}=\mathbb{E}_{w}\left[\frac{\rho^{2}\left(\sum_{l=1}^{L} w_{l}\left(p_{l}-q_{l}\right)\right)^{2}}{\left(1+\rho \sum_{l=1}^{L}\left\{t p_{l}+(1-t) q_{l}\right\} w_{l}\right)^{3}}\right]>0,
$$

which shows convexity. Hence, Theorem 2.1 applies and shows that $\overline{D_{\mathrm{o}}}(\boldsymbol{c})$ is Schur-convex.

An interesting interpretation of the result for the coherent MAC is provided next. In the average distortion expression of the coherent MAC (10), there are two terms which can be identified with well analyzed expressions. The term

$$
\operatorname{EGC}(\boldsymbol{c})=\left(\sum_{l=1}^{L} \sqrt{c_{l} w_{l}}\right)^{2}
$$

can be identified with the SNR after equal gain combining. It is known that this is Schur-convex with respect to $c$ (see [13]). The term

$$
\operatorname{MRC}(\boldsymbol{c})=\left(\sum_{l=1}^{L} c_{l} w_{l}\right)
$$

can be identified with the SNR after maximum ratio combining. This term does not depend on $c$ since

$$
\mathbb{E}_{w}\left(\sum_{l=1}^{L} c_{l} w_{l}\right)=\sum_{l=1}^{L} c_{l}=L
$$

The average distortion can be written as

$$
\overline{D_{\mathrm{c}}}(\boldsymbol{c})=\mathbb{E}_{w}\left[\left(1+\frac{\rho \mathrm{EGC}(\boldsymbol{c})}{2+\rho \mathrm{MRC}(\boldsymbol{c})}\right)^{-1}\right] .
$$

We argue using the results on compositions involving Schurconvex functions in $[9$, Section $3 \mathrm{~B}]$ that the combination in (20) is Schur-convex with respect to $c$.

Worst case sensor placement analysis for orthogonal MAC: The maximum distortion difference due to sensor misplacement is given by the difference of the best case $\left(c_{1}=c_{2}=\right.$ $\left.\ldots=c_{L}=1\right)$ and the worst case $\left(c_{1}=L, c_{2}=c_{3}=\ldots=\right.$ $\left.c_{L}=0\right)$. For the orthogonal MAC, this leads to the expressions

$$
\begin{aligned}
\overline{D_{\mathrm{o}}}(1, \ldots, 1) & =\mathbb{E}_{w}\left[\left(1+\sum_{l=1}^{L}\left(1-\frac{2}{2+\rho w_{l}}\right)\right)^{-1}\right] \\
& =\int_{0}^{\infty} \mathrm{e}^{-t} \prod_{l=1}^{L} \mathbb{E}_{w_{l}}\left[\exp \left(-t\left(1-\frac{2}{2+\rho w_{l}}\right)\right)\right] d t
\end{aligned}
$$

for the best case and

$$
\begin{aligned}
\overline{D_{\mathrm{o}}}([L, 0, \ldots, 0]) & =\mathbb{E}_{w_{1}}\left[\left(1+\left(1-\frac{2}{2+\rho L w_{1}}\right)\right)^{-1}\right] \\
& =\int_{0}^{\infty} \mathrm{e}^{-t} \mathbb{E}_{w_{1}}\left[\exp \left(-t\left(1-\frac{2}{2+\rho L w_{1}}\right)\right)\right] d t
\end{aligned}
$$


for the worst case. For small SNR $\rho \ll 1$, the following approximation for the best case holds

$$
\begin{aligned}
& \overline{D_{\mathrm{o}}}(1, \ldots, 1)=\int_{0}^{\infty} \mathrm{e}^{-t}\left(\mathbb{E}\left[\exp \left(-t\left(1-\frac{2}{2+\rho w_{1}}\right)\right)\right]\right)^{L} d t \\
& \approx \int_{0}^{\infty} \mathrm{e}^{-t}\left(1-\frac{t \rho}{2}\right)^{L} d t=\sum_{k=0}^{L}\left(\begin{array}{l}
L \\
k
\end{array}\right) \int_{0}^{\infty} \mathrm{e}^{-t}\left(-\frac{t \rho}{2}\right)^{k} d t \\
& =\sum_{k=0}^{L}\left(\begin{array}{l}
L \\
k
\end{array}\right)\left(\frac{-\rho}{2}\right)^{k} \Gamma(k+1)
\end{aligned}
$$

where $\Gamma(\cdot)$ is the Gamma function. Considering only the first two terms in the sum in (21), we obtain

$$
\overline{D_{\mathrm{o}}}(1, \ldots, 1) \approx 1-L \frac{\rho}{2}+\mathcal{O}\left(\rho^{2}\right) \geq 1-L \frac{\rho}{2}
$$

For the worst case, we obtain

$$
\overline{D_{\mathrm{o}}}([L, 0, \ldots, 0]) \approx 1-\frac{\rho L}{2} .
$$

Comparing (22) and (23) confirms the result in Theorem 3.1 for small SNR.

\section{B. Random sensor gains, fixed channel gains}

We apply majorization to the average distortion defined in (9) and (10) for the orthogonal and coherent MAC, respectively, in order to show the impact of the average sensor gains.

Theorem 3.2: The average distortion $\overline{D_{\mathrm{o}}}$ in (12) is Schurconvex with respect to average sensor gain vector $\boldsymbol{d}$. This implies that the sensors should be spread out uniformly/symmetrically around the source.

Proof: The average distortion for the orthogonal MAC and random sensor gains can be rewritten as

$\overline{D_{\mathrm{o}}}(\boldsymbol{d})=\mathbb{E}_{\nu}\left[\left(1+\rho \sum_{l=1}^{L}\left(1-\frac{1+\rho}{1+\rho+d_{l} v_{l}}\right)\right)^{-1}\right]$.

This function is symmetric. Using the arguments as in the proof of Theorem 3.1 it is shown that the function is convex. The random variables are interchangeable so we can apply Theorem 2.1 which shows the Schur-convexity of $\overline{D_{\mathrm{o}}}(\boldsymbol{d})$. $\square$

For the average distortion $\overline{D_{\mathrm{c}}}$ in (13) for the coherent MAC, a similar argument as in Section III-A is used to indicate that the average distortion is Schur-convex in this case as well.

\section{Random channels and random sensor gains}

We combine the results from the two previous sections. The average distortion is Schur-convex with respect to $c$ for fixed $\boldsymbol{d}$ and also Schur-convex with respect to $\boldsymbol{d}$ for fixed $\boldsymbol{c}$. The average distortions in (16) and (17) are thus also Schur-convex in the combined vector $\gamma=(\boldsymbol{c}, \boldsymbol{d})$.

For the orthogonal MAC, we make in view of Theorem 3.1 and 3.2 the following observation.

Observation 3.1: The average distortion in (16) is a Schurconvex function with respect to the combined average gain vector $\gamma$. This shows that the sensors should be placed equidistantly from the source and the FC.
The conclusion of Observation 3.1 does not imply that placing all sensor at the same position with equal distance to source and FC is optimal. In the model, we assumed that the channel gains $g_{l}^{2}=c_{l} w_{l}$ and $h_{l}^{2}=d_{l} v_{l}$ have independent small scale fading realizations, i.e., $w_{1}, \ldots, w_{L}$ are i.i.d. and $v_{1}, \ldots, v_{L}$ as well. This shows that there must be a certain distance between sensors to assure independence of the channel and sensor gain realizations. Therefore, the sensor placement in Fig. 3 (black sensors) is optimal for minimum average distortion if the inter-sensor distance is large enough.

\section{OUTAGE DistORTION ANALYSIS}

We briefly point out another performance measure which is important for a slow fading MAC. Define the distortion value $d_{0}$ and $z_{0}=\frac{1}{d_{0}}-1$. The outage probability in the orthogonal $\mathrm{MAC}$ is given by

$$
P_{\text {out }}\left(z_{0}, \boldsymbol{c}\right)=\mathbb{P}\left(\sum_{l=1}^{L} \frac{g_{l}^{2}\left(1-x_{l}\right)}{1 / \rho+g_{l}^{2} x_{l}} \leq z_{0}\right) .
$$

For the fixed sensor gain scenario, i.e., $h_{1}=\ldots=h_{L}=$ 1 , we have $x_{1}=\ldots=x_{L}=1 / 2$. We model $g_{l}^{2}=c_{l} w_{l}$ with i.i.d. standard exponentially distributed random variables $w_{1}, \ldots, w_{L}$. Then it follows that

$$
\sum_{l=1}^{L} \frac{1 / 2 c_{l} w_{l}}{1 / \rho+1 / 2 c_{l} w_{l}}=L-\sum_{l=1}^{L} \frac{2}{2+\rho c_{l} w_{l}}
$$

and the outage probability in (25) can be written as

$$
\begin{aligned}
P_{\text {out }}\left(z_{0}, c\right) & =\mathbb{P}\left(L-\sum_{l=1}^{L} \frac{2}{2+\rho c_{l} w_{l}} \leq z_{0}\right) \\
& =\mathbb{P}(\sum_{l=1}^{L} \frac{2}{2+\rho c_{l} w_{l}} \geq \underbrace{L-z_{0}}_{\xi})
\end{aligned}
$$

Next, an approximation for small SNR $(\rho \ll 1)$ by series expansion is given by

$$
\sum_{l=1}^{L} \frac{2}{2+\rho c_{l} w_{l}}=2-\frac{1}{2}\left(\sum_{l=1}^{L} c_{l} w_{l}\right) \rho+\mathcal{O}\left(\rho^{2}\right) .
$$

Using (27) the outage probability for small SNR is approximated by

$$
P_{\text {out }}\left(z_{0}, \boldsymbol{c}\right) \approx \mathbb{P}\left(\sum_{l=1}^{L} c_{l} w_{l} \leq 2 \cdot \frac{2-\xi}{\rho}\right) .
$$

Invoking Theorem 2.2 leads to the conclusion that for small SNR, i.e. for a RHS of (28) larger than two, the outage probability is Schur-concave with respect to $c$. This behavior is illustrated in the second example of the next section.

\section{ILLUSTRATIONS}

In Fig. 4, the average distortion for the orthogonal and the coherent MAC with two sensors and fixed sensor gains $h_{1}=$ $h_{2}=1$ is shown for different average channel gains of sensor $S_{1}$. Since the total average channel gain is constant, we have $c_{2}=1-c_{1}$. Note that the symmetric equidistant scenario, i.e. $c_{1}=c_{2}=\frac{1}{2}$, should have lowest average distortion. 


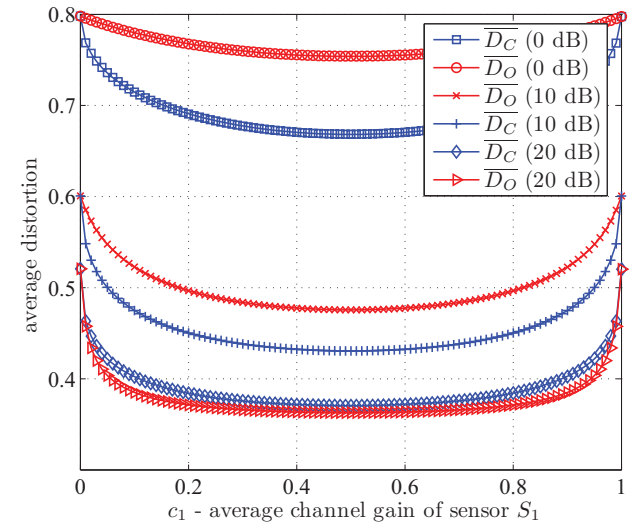

Fig. 4. Average distortion as function of SNR and average channel gain for two sensors.
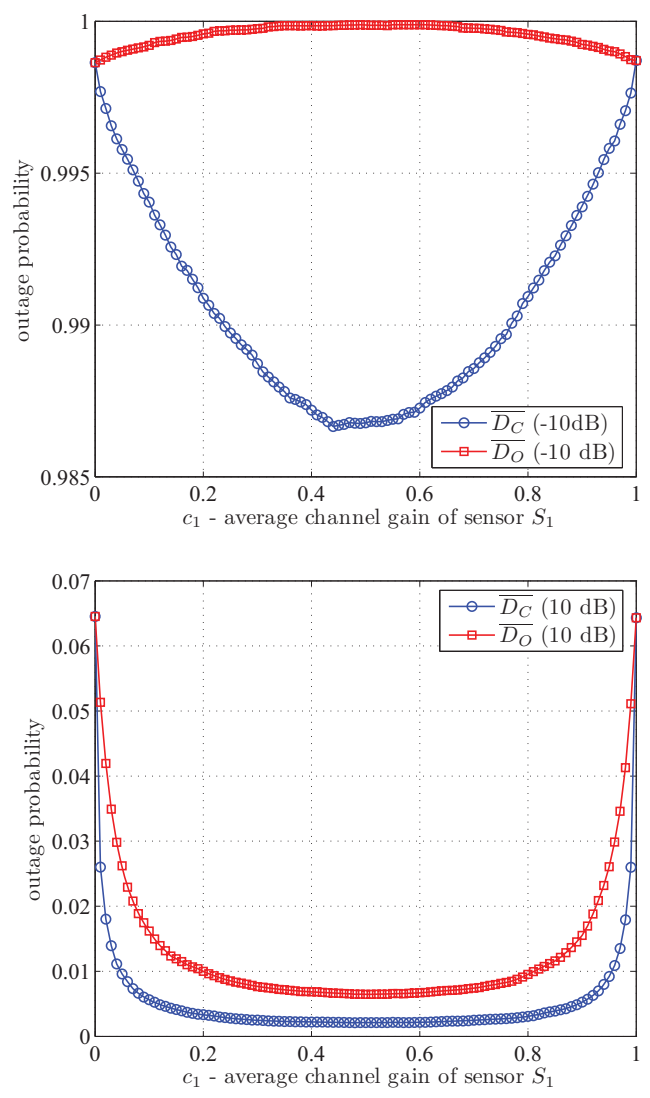

Fig. 5. Outage probability of the distortion for $d_{0}=0.8$ as function of SNR and average channel gain for two sensors.

Fig. 4 illustrates the result of Theorem 3.1 and shows also that for high SNR, the orthogonal MAC outperforms the coherent MAC (see equations (7) and (8)).

Fig. 5 shows the outage probability for the distortion for the orthogonal MAC for two sensors with different average channel gains and SNR. For high SNR, the behavior is identical to the average distortion and symmetric sensor placement yields minimum outage probability. However, for small SNR and admittedly incredible high outage probabilities, the behavior turns around and an asymmetric sensor placements gives lower outage probability. Interestingly, the behavior for the coherent MAC seems to be consistent for small and high SNR values.

\section{CONCLUSIONS AND OPEN PROBLEMS}

In order to minimize the average distortion using an orthogonal or coherent MAC, it is optimal to place the sensors equidistantly between source and FC, e.g., on a circle with inter-sensor distance larger than two times the wavelength but small inter-sensor distance compared to the overall distance to source and FC. This conclusion changes theoretically if the outage probability is applied as the performance measure. Practically, the outage probability is also Schur-convex as a function of the average channel gains for all reasonable outage probabilities.

In this work, we have assumed that the sensors have individual power constraints. If this assumption is relaxed, the above conclusion might change and an asymmetric sensor distribution could achieve lower distortion. Another direction for future research is to consider multiple sources.

\section{REFERENCES}

[1] I. Oppermann, L. Stoica, A. Rabbachin, Z. Shelby, and J. Haapola, "UWB wireless sensor networks: UWEN - a practical example," IEEE Communications Magazine, vol. 42, no. 12, pp. 27-32, Dec. 2004.

[2] W. Bajwa, A. Sayeed, and R. Nowak, "Matched source-channel communication for field estimation in wireless sensor network," in Proc. of 4th International Symposium on Information Processing in Sensor Networks., April 2005, pp. 332-339.

[3] J.-J. Xiao, S. Cui, Z.-Q. Luo, and A. J. Goldsmith, "Linear coherent decentralized estimation," IEEE Trans. on Signal Processing, vol. 56, pp. 757-770, 2008.

[4] J. Li, L. L. Andrew, C. H. Foh, M. Zukerman, and H.-H. Chen, "Connectivity, coverage and placement in wireless sensor networks," Sensors, vol. 9, no. 10, pp. 7664-7693, 2009.

[5] A. Kumar, P. Ishwa, and K. Ramchandran, "High-resolution distributed sampling of bandlimited fields with low-precision sensors," IEEE Trans. on Information Theory, submitted, 2008.

[6] A. Marques, X. Wang, and G. Giannakis, "Minimizing transmit power for coherent communications in wireless sensor networks with finiterate feedback," IEEE Trans. on Signal Processing,, vol. 56, no. 9, pp. 4446-4457, Sept. 2008.

[7] A. D. Sarwate and M. Gastpar, "Fading observation alignment via feedback," in Proc. of 4th International Symposium on Information Processing in Sensor Networks, 2005.

[8] J. N. Tsitsiklis and Z.-Q. Luo, "Communication complexity of convex optimization," Journal of Complexity, vol. 3, pp. 231-243, 1987.

[9] A. W. Marshall and I. Olkin, Inequalities: Theory of Majorization and Its Application. Mathematics in Science and Engineering vol. 143, Academic Press, Inc. (London) Ltd., 1979.

[10] E. A. Jorswieck and H. Boche, Majorization and Matrix Monotone Functions in Wireless Communications, ser. Foundations and Trends in Communications and Information Theory. Now publishers, 2007, vol. 3.

[11] A. W. Marshall and F. Proschan, "An inequality for convex functions involving majorization," Journal of Mathematical Analysis and Applications, 1965.

[12] E. A. Jorswieck and H. Boche, "Outage probability in multiple antenna systems," European Trans. on Telecommunications, vol. 18, pp. 217233, 2007.

[13] E. A. Jorswieck, T. Oechtering, and H. Boche, "Performance analysis of combining techniques with correlated diversity," Proc. of IEEE Wireless Communications and Networking Conference, vol. 2, pp. 849-854, 2005. 